\title{
ON THE DETERIORATION OF NONREPAIRABLE MULTISTATE STRONGLY COHERENT SYSTEMS
}

\author{
BENT NATVIG,* University of Oslo
}

\begin{abstract}
In the present paper, results given in Natvig (1990) are generalized to a multistate, strongly coherent, nonrepairable system of independent components by considering the reduction in remaining system time above a certain state due to a jump downwards of a component. This reduction also equals the increase in remaining system time above a certain state due to a minimal repair of the component at its time of jump downwards. The expected value of the sum of such reductions/increases for the different possible jumps downwards of the component is the building block of the Natvig measure of the importance of the component in the multistate case. Hence, the whole distributions of these reductions/increases are arrived at, not only the expectations, throwing more light on the consequences for the system of the deterioration of the components.
\end{abstract}

Keywords: Dynamic reliability; Natvig importance of a system component; multistate strongly coherent system; nonrepairable system

2010 Mathematics Subject Classification: Primary 62N05; 90B25

\section{Introduction}

Natvig (1990) considered the reduction in remaining system lifetime due to the failure of a specific component and a specific module in a binary, coherent, nonrepairable system of independent components. The former reduction also equals the increase in remaining system lifetime due to a minimal repair of the component at its time of failure. The expected value of this reduction/increase is the building block of the so-called Natvig measure of the importance of the component as treated in Natvig (1979), (1982a), (1985), and Natvig and Gåsemyr (2009). In Natvig (1990) the whole distribution of this reduction/increase was derived, not just the expectation. In the present paper these results are generalized to a multistate, strongly coherent, nonrepairable system of independent components by considering the reduction in remaining system time above a certain state due to a jump downwards of a component. Again, this reduction also equals the increase in remaining system time above a certain state due to a minimal repair of the component at its time of jump downwards. The expected value of the sum of such reductions/increases for the different possible jumps downwards of the component is the building block of the Natvig measure of the importance of the component in the multistate case as treated in Natvig (2011a). Here, the whole distributions of these reductions/increases are arrived at, not only the expectations, throwing more light on the consequences for the system of the deterioration of the components.

Examples of contributions to the area of component importance in multistate systems are presented in Ramirez-Marquez et al. (2006), Ramirez-Marquez and Coit (2007), Zio, Podofillini and Levitin (2004), and Zio, Marella and Podofillini (2007). The latter gives an application to

Received 4 September 2012; revision received 25 April 2013.

* Postal address: Department of Mathematics, University of Oslo, PO Box 1053 Blindern, Oslo, N-0316, Norway.

Email address: bent@math.uio.no 
the railway industry. Furthermore, a part of the recent book Kuo and Zhu (2012) is devoted to this area.

Let $S=\{0,1, \ldots, M\}$ be the set of states of the system; the $M+1$ states represent successive levels of performance ranging from the perfect functioning level $M$ down to the complete failure level 0 . Furthermore, let $C=\{1, \ldots, n\}$ be the set of components and, in general, $S_{i}$, $i=1, \ldots, n$, the set of states of the $i$ th component. We claim $\{0, M\} \subseteq S_{i} \subseteq S$. Hence, the states 0 and $M$ are chosen to represent the endpoints of a performance scale that might be used for both the system and its components. Note that in most applications there is no need for the same detailed description of the components as for the system.

Let $x_{i}, i=1, \ldots, n$, denote the state or performance level of the $i$ th component at a fixed point of time and $\boldsymbol{x}=\left(x_{1}, \ldots, x_{n}\right)$. It is assumed that the state, $\phi$, of the system at the fixed point of time is a deterministic function of $\boldsymbol{x}$; i.e. $\phi=\phi(\boldsymbol{x})$. Here $\boldsymbol{x}$ takes values in $S_{1} \times S_{2} \times \cdots \times S_{n}$ and $\phi$ takes values in $S$. The function $\phi$ is called the structure function of the system. We often denote a multistate system by $(C, \phi)$.

Definition 1. A system is a multistate monotone system (MMS) if and only if its structure function $\phi$ satisfies the following properties:

(i) $\phi(\boldsymbol{x})$ is nondecreasing in each argument,

(ii) $\phi(\mathbf{0})=0$ and $\phi(\boldsymbol{M})=M$, where $\mathbf{0}=(0, \ldots, 0), \boldsymbol{M}=(M, \ldots, M)$.

Let $(\cdot i, \boldsymbol{x})=\left(x_{1}, \ldots, x_{i-1}, \cdot, x_{i+1}, \ldots, x_{n}\right)$. Now choose $j \in\{1, \ldots, M\}$ and let the states $\{0, \ldots, j-1\}$ correspond to the failure state and $\{j, \ldots, M\}$ to the functioning state if a binary approach had been applied. Following this approach it seems natural, for any way of distinguishing between the binary failure and functioning state, to claim each component to be relevant. More precisely, for any $j \in\{1, \ldots, M\}$ and any component $i$ there should exist a vector $\left({ }_{i}, \boldsymbol{x}\right)$ such that if the $i$ th component is in the binary failure state, the system itself is in the binary failure state, and, correspondingly, if the $i$ th component is in the binary functioning state, the system itself is in the binary functioning state. This motivates the following definition of a multistate strongly coherent system, which, for the case $S_{i}=S, i=1, \ldots, n$, is introduced as a multistate coherent system of type 1 in Natvig (1982b).

The following notation is needed

$$
S_{i, j}^{0}=S_{i} \cap\{0, \ldots, j-1\} \quad \text { and } \quad S_{i, j}^{1}=S_{i} \cap\{j, \ldots, M\} .
$$

Definition 2. Consider an MMS with structure function $\phi$ satisfying

$$
\min _{1 \leq i \leq n} x_{i} \leq \phi(\boldsymbol{x}) \leq \max _{1 \leq i \leq n} x_{i}
$$

where $\min _{1 \leq i \leq n} x_{i}$ and $\max _{1 \leq i \leq n} x_{i}$ are respectively the multistate series and parallel structure functions. If, in addition, for all $i \in\{1, \ldots, n\}$, for all $j \in\{1, \ldots, M\}$, there exists $\left({ }_{i}, \boldsymbol{x}\right)$ such that

$$
\phi\left(k_{i}, \boldsymbol{x}\right) \geq j, \quad \phi\left(\ell_{i}, \boldsymbol{x}\right)<j, \quad \text { for all } k \in S_{i, j}^{1} \text { and } \ell \in S_{i, j}^{0},
$$

we have a multistate strongly coherent system (MSCS).

In this paper we will concentrate on multistate strongly coherent systems. We now consider the relation between the stochastic performance of the system $(C, \phi)$ and the stochastic performances of the components. We introduce the random state $X_{i}(t)$ of the $i$ th component at time $t, i=1, \ldots, n$, and the corresponding random vector $X(t)=\left(X_{1}(t), \ldots, X_{n}(t)\right)$. Now, if $\phi$ is a multistate structure function, $\phi(X(t))$ is the corresponding random system state at time $t$. 
Assume also that the stochastic processes $\left\{X_{i}(t), t \in[0, \infty)\right\}, i=1, \ldots, n$, are mutually independent. For the dynamic approach of the present paper this is a necessary assumption in order to arrive at explicit results.

We restrict our attention to the case where the components, and hence the system, cannot be repaired. In order to avoid a rather complex notation we will in the following assume that $S_{i}=S, i=1, \ldots, n$. Furthermore, assume that at time $t=0$ all components are in the perfect functioning state $M$; i.e. $\boldsymbol{X}(0)=\boldsymbol{M}$. We introduce the notation

$$
\begin{gathered}
\mathbb{P}\left(X_{i}(t)=j\right)=r_{i}^{j}(t), \quad j=0, \ldots, M, \\
\boldsymbol{r}(t)=\left(r_{1}^{1}(t), \ldots, r_{1}^{M}(t), r_{2}^{1}(t), \ldots, r_{n}^{M}(t)\right), \\
p_{i}^{k, \ell}(t, t+u)=\mathbb{P}\left(X_{i}(t+u)=\ell \mid X_{i}(t)=k\right), \quad 0 \leq \ell<k \leq M, \\
\lambda_{i}^{k, \ell}(t)=\lim _{h \rightarrow 0} \frac{p_{i}^{(k, \ell)}(t, t+h)}{h}, \quad 0 \leq \ell<k \leq M, \\
\mathbb{P}(\phi(\boldsymbol{X}(t)) \geq j)=\mathbb{P}(\mathbf{1}(\phi(\boldsymbol{X}(t)) \geq j)=1)=p_{\phi}^{j}(\boldsymbol{r}(t)),
\end{gathered}
$$

where $\mathbf{1}(\cdot)$ is the indicator function and $p_{\phi}^{j}(\boldsymbol{r}(t))$ is the reliability to level $j$ of the system at time $t$.

In order to make things not too complex we assume that

$$
\lambda_{i}^{k, \ell}(t)=0, \quad 0 \leq \ell<k-1 \leq M-1 .
$$

Hence, each component deteriorates by going through all states from the perfect functioning state until the complete failure state. Let the $i$ th component have an absolutely continuous distribution $F_{i}^{k}(t)$ of time spent in state $k$, before jumping downwards to state $k-1$, with density $f_{i}^{k}(t)$ and $\bar{F}_{i}^{k}(t)=1-F_{i}^{k}(t)$. It is assumed that all these times spent in the various states are independent. Hence, $X_{i}(t), i=1, \ldots, n$, for $t \in[0, \infty)$, are semi-Markov processes in continuous time, not Markov processes as stated in Natvig (2011a), (2011b).

The paper is organized as follows. In Section 2 we consider the reduction in remaining system time above state $j \in\{1, \ldots, M\}$ due to a jump downwards of the $i$ th component from state $k \in\{1, \ldots, M\}$. Corresponding results for a module are given in Section 3. Finally, some concluding remarks are given in Section 4.

\section{Reduction in the remaining system time above a specific state due to a jump downwards of a component}

Intuitively, it seems that components that strongly reduce the expected remaining system time in the better states by deteriorating are very important. This seems at least true during the system development phase. This is the motivation for the following generalization to multistate systems, given in Natvig (2011a), of the Natvig (1979) measure of the importance of the $i$ th component. In Natvig (2011a), as in the binary case treated in Natvig (1982a), the actual definition of this generalized measure is in terms of the effect on future system performance of a fictive minimal repair of a component.

We introduce, for $i=1, \ldots, n, k \in\{0, \ldots, M-1\}$,

- $T_{i, k}$, the time of the jump of the $i$ th component into state $k$,

- $T_{i, k}^{\prime}$, the fictive time of the jump of the $i$ th component into state $k$ after a fictive minimal repair of the component at $T_{i, k}$; i.e. it is repaired to have the same distribution of remaining time in state $k+1$ as it had just before jumping downwards to state $k$. 
Furthermore, for $i=1, \ldots, n, k \in\{1, \ldots, M\}$, and $j \in\{1, \ldots, M\}$, we introduce

$$
Z_{i, k, j}=Y_{i, k, j}^{1}-Y_{i, k, j}^{0}
$$

where

- $Y_{i, k, j}^{1}$ is the system time in the states $\{j, \ldots, M\}$ in the interval $\left[T_{i, k-1}, T_{i, k-1}^{\prime}\right]$ just after the jump downwards from state $k$ to state $k-1$ of the $i$ th component, which, however, immediately undergoes a fictive minimal repair,

- $Y_{i, k, j}^{0}$ is the system time in the states $\{j, \ldots, M\}$ in the interval $\left[T_{i, k-1}, T_{i, k-1}^{\prime}\right]$ just after the jump downwards from state $k$ to state $k-1$ of the $i$ th component, assuming that the component stays in the latter state throughout this interval.

Thus, $Z_{i, k, j}$ is the fictive increase in system time in the states $\{j, \ldots, M\}$ in the interval $\left[T_{i, k-1}, T_{i, k-1}^{\prime}\right]$ due to a fictive minimal repair of the $i$ th component when jumping downwards from state $k$ to state $k-1$. Note that since the minimal repair is fictive, we have chosen to calculate the effect of this repair over the entire interval $\left[T_{i, k-1}, T_{i, k-1}^{\prime}\right]$ even though this interval may extend beyond the time of the next jump of the $i$ th component. Note that the fictive minimal repair periods, i.e. the intervals of the form $\left[T_{i, k-1}, T_{i, k-1}^{\prime}\right]$, may sometimes overlap. Thus, at a given point of time we may have contributions from more than one fictive minimal repair. This was efficiently dealt with by the simulation methods presented in Huseby and Natvig (2013). Taking the expectation, we obtain, for $i=1, \ldots, n$ and $j \in\{1, \ldots, M\}$, the following generalized Natvig measure, $I_{N}^{(i, j)}$, of the importance of the $i$ th component

$$
I_{N}^{(i, j)}=\frac{\sum_{k=1}^{M} \mathbb{E} Z_{i, k, j}}{\sum_{r=1}^{n} \sum_{k=1}^{M} \mathbb{E} Z_{r, k, j}},
$$

tacitly assuming that $\mathbb{E} Z_{i, k, j}<\infty$ for $i=1, \ldots, n, k \in\{1, \ldots, M\}$, and $j \in\{1, \ldots, M\}$. We obviously have

$$
\sum_{i=1}^{n} I_{N}^{(i, j)}=1, \quad 0 \leq I_{N}^{(i, j)} \leq 1 .
$$

Lemmas 1 and 2 below are given here without proofs except for noting that the terms $\left(\bar{F}_{i}^{k}(z+u) / \bar{F}_{i}^{k}(z)\right)$ and $\left(\bar{F}_{i}^{k}(z+v) / \bar{F}_{i}^{k}(v)\right)$ in Lemma 1 are entering since we are considering the interval $\left[T_{i, k-1}, T_{i, k-1}^{\prime}\right]$. These lemmas are generalizations of Lemma 2.1 in Natvig (1982a) covering a binary coherent system.

Lemma 1. For $k \in\{1, \ldots, M-1\}$,

$$
\begin{aligned}
\bar{G}_{i, k, j}^{1}(u)= & \mathbb{P}\left(Y_{i, k, j}^{1}>u\right) \\
= & \int_{s_{i}=0}^{\infty} \int_{z=0}^{\infty} p_{\phi}^{j}\left(\left(\mathbf{0}, 1_{k}\right)_{i}, \boldsymbol{r}\left(s_{i}+z+u\right)\right) r_{i}^{k+1}\left(s_{i}\right) \lambda_{i}^{k+1, k}\left(s_{i}\right) f_{i}^{k}(z) \\
& \quad \times \frac{\bar{F}_{i}^{k}(z+u)}{\bar{F}_{i}^{k}(z)} \mathrm{d} z \mathrm{~d} s_{i}, \\
\bar{G}_{i, M, j}^{1}(u)= & \mathbb{P}\left(Y_{i, M, j}^{1}>u\right) \\
= & \int_{0}^{\infty} p_{\phi}^{j}\left(\left(\mathbf{0}, 1_{M}\right)_{i}, \boldsymbol{r}(z+u)\right) f_{i}^{M}(z) \frac{\bar{F}_{i}^{M}(z+u)}{\bar{F}_{i}^{M}(z)} \mathrm{d} z,
\end{aligned}
$$




$$
\begin{aligned}
\bar{G}_{i, k, j}^{0}(v)= & \mathbb{P}\left(Y_{i, k, j}^{0}>v\right) \\
= & \int_{s_{i}=0}^{\infty} \int_{z=0}^{\infty} p_{\phi}^{j}\left(\left(\mathbf{0}, 1_{k-1}\right)_{i}, \boldsymbol{r}\left(s_{i}+z+v\right)\right) r_{i}^{k+1}\left(s_{i}\right) \lambda_{i}^{k+1, k}\left(s_{i}\right) f_{i}^{k}(z) \\
& \quad \times \frac{\bar{F}_{i}^{k}(z+v)}{\bar{F}_{i}^{k}(z)} \mathrm{d} z \mathrm{~d} s_{i}, \\
\bar{G}_{i, M, j}^{0}(v)= & \mathbb{P}\left(Y_{i, M, j}^{0}>v\right) \\
= & \int_{0}^{\infty} p_{\phi}^{j}\left(\left(\mathbf{0}, 1_{M-1}\right)_{i}, \boldsymbol{r}(z+v)\right) f_{i}^{M}(z) \frac{\bar{F}_{i}^{M}(z+v)}{\bar{F}_{i}^{M}(z)} \mathrm{d} z .
\end{aligned}
$$

From Lemma 1 we arrive at the following expression for the probability that the jump downwards of the $i$ th component from state $k \in\{1, \ldots, M-1\}$ to state $k-1$ leads to the system leaving the states $\{j, \ldots, M\}$.

$$
\begin{aligned}
\mathbb{P}\left(Y_{i, k, j}^{1}>0, Y_{i, k, j}^{0}=0\right)= & \mathbb{P}\left(Y_{i, k, j}^{0}=0\right)-\mathbb{P}\left(Y_{i, k, j}^{1}=0, Y_{i, k, j}^{0}=0\right) \\
= & \mathbb{P}\left(Y_{i, k, j}^{0}=0\right)-\mathbb{P}\left(Y_{i, k, j}^{1}=0\right) \\
= & \bar{G}_{i, k, j}^{1}(0)-\bar{G}_{i, k, j}^{0}(0) \\
= & \int_{s_{i}=0}^{\infty} \int_{z=0}^{\infty} p_{\phi}^{j}\left(\left(\mathbf{0}, 1_{k}\right)_{i}, \boldsymbol{r}\left(s_{i}+z\right)\right) r_{i}^{k+1}\left(s_{i}\right) \lambda_{i}^{k+1, k}\left(s_{i}\right) f_{i}^{k}(z) \mathrm{d} z \mathrm{~d} s_{i} \\
& -\int_{s_{i}=0}^{\infty} \int_{z=0}^{\infty} p_{\phi}^{j}\left(\left(\mathbf{0}, 1_{k-1}\right), r\left(s_{i}+z\right)\right) \\
= & \int_{s_{i}=0}^{\infty} \int_{z=0}^{\infty} I_{B}^{(i, k, j)}\left(s_{i}^{k+1}+z\right) r_{i}^{k+1}\left(s_{i}\right) \lambda_{i}^{k+1, k}\left(s_{i}\right) f_{i}^{k}(z) \mathrm{d} z \mathrm{~d} s_{i} \\
= & \left.\int_{0}^{\infty} I_{B}^{(i, k, j)}(t) r_{i}^{k}\right) f_{i}^{k}(z) \lambda_{i}^{k, k-1}(t) \mathrm{d} z,
\end{aligned}
$$

where

$$
I_{B}^{(i, k, j)}(t)=p_{\phi}^{j}\left(\left(\mathbf{0}, 1_{k}\right)_{i}, \boldsymbol{r}(t)\right)-p_{\phi}^{j}\left(\left(\mathbf{0}, 1_{k-1}\right)_{i}, \boldsymbol{r}(t)\right) .
$$

The same expressions follow easily for $k=M$. The latter expression is the generalized Birnbaum measure (Birnbaum (1969)) of the importance of the $i$ th component at time $t$ given in Natvig (2011a). This being the probability that the system is in one of the states $\{j, \ldots, M\}$ if at time $t$ the $i$ th component is in state $k$ and not if the $i$ th component is in state $k-1$. By summing (1) from $k=1$ to $k=M$ we arrive at the generalized Barlow-Proschan measure (Barlow and Proschan (1975)) of the importance of the $i$ th component given in Natvig (2011a). This being the probability that the jump downwards of the $i$ th component coincides with the system leaving the states $\{j, \ldots, M\}$.

Note also that the probability that the system is leaving the states $\{j, \ldots, M\}$ before the jump downwards of the $i$ th component from state $k \in\{1, \ldots, M\}$ to state $k-1$ is given by

$$
\mathbb{P}\left(Y_{i, k, j}^{1}=0\right)=1-\bar{G}_{i, k, j}^{1}(0)=1-\int_{0}^{\infty} p_{\phi}^{j}\left(\left(\mathbf{0}, 1_{k}\right)_{i}, \boldsymbol{r}(t)\right) r_{i}^{k}(t) \lambda_{i}^{k, k-1}(t) \mathrm{d} t .
$$


Lemma 2. For $k \in\{1, \ldots, M-1\}, 0 \leq v \leq u$,

$$
\begin{aligned}
\bar{G}_{i, k, j}(u, v)= & \mathbb{P}\left(Y_{i, k, j}^{1}>u, Y_{i, k, j}^{0}>v\right) \\
= & \int_{s_{i}=0}^{\infty} \int_{z=0}^{\infty} \sum_{\left(\cdot{ }_{i}, \boldsymbol{x}\right)\left({ }_{\cdot}, \boldsymbol{y}\right) \leq\left(\cdot_{i}, \boldsymbol{x}\right)} \sum \mathbf{1}\left(\phi\left((k-1)_{i}, \boldsymbol{x}\right) \geq j\right) \mathbf{1}\left(\phi\left(k_{i}, \boldsymbol{y}\right) \geq j\right) \\
& \times \prod_{l \neq i}\left[r_{l}^{x_{l}}\left(s_{i}+z+v\right) p_{l}^{x_{l}, y_{l}}\left(s_{i}+z+v, s_{i}+z+u\right)\right] \\
& \times r_{i}^{k+1}\left(s_{i}\right) \lambda_{i}^{k+1, k}\left(s_{i}\right) f_{i}^{k}(z) \frac{\bar{F}_{i}^{k}(z+u)}{\bar{F}_{i}^{k}(z)} \mathrm{d} z \mathrm{~d} s_{i}, \\
\bar{G}_{i, M, j}(u, v)= & \mathbb{P}\left(Y_{i, M, j}^{1}>u, Y_{i, M, j}^{0}>v\right) \\
= & \left.\int_{z=0}^{\infty} \sum_{\left({ }_{i}, \boldsymbol{x}\right)} \sum_{\left({ }_{i}, \boldsymbol{y}\right) \leq\left({ }_{i}, \boldsymbol{x}\right)} \mathbf{1}\left(\phi((M-1))_{i}, \boldsymbol{x}\right) \geq j\right) \mathbf{1}\left(\phi\left(M_{i}, \boldsymbol{y}\right) \geq j\right) \\
& \times \prod_{l \neq i}\left[r_{l}^{x_{l}}(z+v) p_{l}^{x_{l}, y_{l}}(z+v, z+u)\right] f_{i}^{M}(z) \frac{\bar{F}_{i}^{M}(z+u)}{\bar{F}_{i}^{M}(z)} \mathrm{d} z .
\end{aligned}
$$

The distribution of $Z_{i, k, j}$ is given by the following theorem. The proof for $\mathbb{P}\left(Z_{i, k, j}=0\right)$ follows partly from (1). The proof for $\mathbb{P}\left(Y_{i, k, j}^{1}>Y_{i, k, j}^{0}>0\right)$ is not based on minimal cut sets containing the $i$ th component, as used in Natvig (1990) which treated the binary case, since these sets may be identical in the multistate case even if the minimal cut vectors are different. The proof for the absolutely continuous part is completely parallel to the one given in Theorem 2.3 of Natvig (1982a) but now inserting the expressions for $\bar{G}_{i, k, j}^{1}(u)$ and $\bar{G}_{i, k, j}(u, v)$ from Lemma 1 and 2.

Theorem 1. For $k \in\{1, \ldots, M-1\}$,

$$
\begin{aligned}
& \mathbb{P}\left(Z_{i, k, j}=0\right) \\
& =1-\mathbb{P}\left(Z_{i, k, j}>0\right) \\
& =1-\mathbb{P}\left(Y_{i, k, j}^{1}>Y_{i, k, j}^{0}>0\right)-\mathbb{P}\left(Y_{i, k, j}^{1}>0, Y_{i, k, j}^{0}=0\right) \\
& =1-\left[\int_{s_{i}=0}^{\infty} \int_{z=0}^{\infty} \int_{v=0}^{\infty} r_{i}^{k+1}\left(s_{i}\right) \lambda_{i}^{k+1, k}\left(s_{i}\right) f_{i}^{k}(z) \frac{\bar{F}_{i}^{k}(z+v)}{\bar{F}_{i}^{k}(z)}\right. \\
& \times \sum_{(\cdot i, \boldsymbol{x})} \prod_{h \neq i} r_{h}^{x_{h}}\left(s_{i}+z+v\right) \\
& \times \sum_{l \neq i} \mathbf{1}\left(x_{l}>0\right) \lambda_{l}^{x_{l}, x_{l}-1}\left(s_{i}+z+v\right) \\
& \times\left[\mathbf{1}\left(\phi\left((k-1)_{i},\left(x_{l}\right)_{l}, \boldsymbol{x}\right) \geq j\right)\right. \\
& \times \mathbf{1}\left(\phi\left(k_{i},\left(x_{l}-1\right)_{l}, \boldsymbol{x}\right) \geq j\right) \\
& \left.\left.-\mathbf{1}\left(\phi\left((k-1)_{i},\left(x_{l}-1\right)_{l}, \boldsymbol{x}\right) \geq j\right)\right] \mathrm{d} v \mathrm{~d} z \mathrm{~d} s_{i}\right] \\
& -\int_{0}^{\infty} I_{B}^{(i, k, j)}(t) r_{i}^{k}(t) \lambda_{i}^{k, k-1}(t) \mathrm{d} t,
\end{aligned}
$$




$$
\begin{gathered}
\mathbb{P}\left(Z_{i, M, j}=0\right) \\
=1-\mathbb{P}\left(Z_{i, M, j}>0\right) \\
=1-\mathbb{P}\left(Y_{i, M, j}^{1}>Y_{i, M, j}^{0}>0\right)-\mathbb{P}\left(Y_{i, M, j}^{1}>0, Y_{i, M, j}^{0}=0\right) \\
=1-\left[\int_{z=0}^{\infty} \int_{v=0}^{\infty} f_{i}^{M}(z) \frac{\bar{F}_{i}^{M}(z+v)}{\bar{F}_{i}^{M}(z)}\right. \\
\times \sum_{(\cdot i, \boldsymbol{x})} \prod_{h \neq i} r_{h}^{x_{h}}(z+v) \\
\times \sum_{l \neq i} \mathbf{1}\left(x_{l}>0\right) \lambda_{l}^{x_{l}, x_{l}-1}(z+v) \\
\times\left[\mathbf{1}\left(\phi\left((M-1)_{i},\left(x_{l}\right)_{l}, \boldsymbol{x}\right) \geq j\right)\right. \\
\times \mathbf{1}\left(\phi\left(M_{i},\left(x_{l}-1\right)_{l}, \boldsymbol{x}\right) \geq j\right) \\
\left.\left.-\mathbf{1}\left(\phi\left((M-1)_{i},\left(x_{l}-1\right)_{l}, \boldsymbol{x}\right) \geq j\right)\right] \mathrm{d} v \mathrm{~d} z\right] \\
-\int_{0}^{\infty} I_{B}^{(i, M, j)}(t) r_{i}^{M}(t) \lambda_{i}^{M, M-1}(t) \mathrm{d} t .
\end{gathered}
$$

Furthermore, let

$$
\begin{gathered}
g_{i, k, j}(u, v)=\frac{\partial^{2} \bar{G}_{i, k, j}(u, v)}{\partial u \partial v}, \quad 0<v<u, \\
g_{i, k, j}^{1}(u, 0)=\frac{\partial\left[\bar{G}_{i, k, j}(u, 0)-\bar{G}_{i, k, j}^{1}(u)\right]}{\partial u}, \quad 0<u .
\end{gathered}
$$

Then the absolutely continuous part of the distribution of $Z_{i, k, j}$ has density

$$
g_{i, k, j}(z)=g_{i, k, j}^{1}(z, 0)+\int_{0}^{\infty} g_{i, k, j}(v+z, v) \mathrm{d} v, \quad 0<z .
$$

To illustrate the theory we now consider the multistate series and parallel systems as given in Natvig (2011b) generalizing results from Natvig (1982a) covering the binary case. This is first shown for the multistate series system $\phi(x)=\min _{1 \leq i \leq n} x_{i}$. We consider the case $k=j$ for $j \in\{1, \ldots, M-1\}$. Now obviously $Z_{i, j, j}=Y_{i, j, j}^{1}$. From Lemmas 1 and 2 we have

$$
\begin{gathered}
\bar{G}_{i, j, j}^{1}(u)=\int_{s_{i}=0}^{\infty} \int_{z=0}^{\infty} \prod_{l \neq i} \sum_{m=j}^{M} r_{l}^{m}\left(s_{i}+z+u\right) r_{i}^{j+1}\left(s_{i}\right) \lambda_{i}^{j+1, j}\left(s_{i}\right) f_{i}^{j}(z) \frac{\bar{F}_{i}^{j}(z+u)}{\bar{F}_{i}^{j}(z)} \mathrm{d} z \mathrm{~d} s_{i}, \\
\bar{G}_{i, j, j}^{0}(v)=0, \\
\bar{G}_{i, j, j}(u, v)=0 .
\end{gathered}
$$

From Theorem 1 we obtain

$$
\mathbb{P}\left(Z_{i, j, j}=0\right)=1-\int_{0}^{\infty} \prod_{l \neq i} \sum_{m=j}^{M} r_{l}^{m}(t) r_{i}^{j}(t) \lambda_{i}^{j, j-1}(t) \mathrm{d} t .
$$


We now turn to the multistate parallel system where $\phi(x)=\max _{1 \leq i \leq n} x_{i}$. Again, we consider the case $k=j$ for $j \in\{1, \ldots, M-1\}$ and introduce the notation

$$
\coprod_{l \in A} x_{l}=1-\prod_{l \in A}\left(1-x_{l}\right) .
$$

From Lemmas 1 and 2 we have

$$
\begin{gathered}
\bar{G}_{i, j, j}^{1}(u)=\int_{s_{i}=0}^{\infty} \int_{z=0}^{\infty} r_{i}^{j+1}\left(s_{i}\right) \lambda_{i}^{j+1, j}\left(s_{i}\right) f_{i}^{j}(z) \frac{\bar{F}_{i}^{j}(z+u)}{\bar{F}_{i}^{j}(z)} \mathrm{d} z \mathrm{~d} s_{i}, \\
\bar{G}_{i, j, j}^{0}(v)=\int_{s_{i}=0}^{\infty} \int_{z=0}^{\infty} \coprod_{l \neq i} \sum_{m=j}^{M} r_{l}^{m}\left(s_{i}+z+v\right) r_{i}^{j+1}\left(s_{i}\right) \lambda_{i}^{j+1, j}\left(s_{i}\right) f_{i}^{j}(z) \frac{\bar{F}_{i}^{j}(z+v)}{\bar{F}_{i}^{j}(z)} \mathrm{d} z \mathrm{~d} s_{i}, \\
\bar{G}_{i, j, j}(u, v)=\int_{s_{i}=0}^{\infty} \int_{z=0}^{\infty} \sum_{(\cdot i, x)} \coprod_{h \neq i} \mathbf{1}\left(x_{h} \geq j\right) \prod_{l \neq i} r_{l}^{x_{l}}\left(s_{i}+z+v\right) \\
\times r_{i}^{j+1}\left(s_{i}\right) \lambda_{i}^{j+1, j}\left(s_{i}\right) f_{i}^{j}(z) \frac{\bar{F}_{i}^{j}(z+u)}{\bar{F}_{i}^{j}(z)} \mathrm{d} z \mathrm{~d} s_{i} .
\end{gathered}
$$

From Theorem 1 we obtain

$$
\begin{gathered}
\mathbb{P}\left(Z_{i, j, j=0)=1}-\int_{s_{i}=0}^{\infty} \int_{z=0}^{\infty} \int_{v=0}^{\infty} r_{i}^{j+1}\left(s_{i}\right) \lambda_{i}^{j+1, j}\left(s_{i}\right) f_{i}^{j}(z) \frac{\bar{F}_{i}^{j}(z+v)}{\bar{F}_{i}^{j}(z)}\right. \\
\times \sum_{l \neq i} \prod_{h \neq i, l} \sum_{m=0}^{j-1} r_{h}^{m}\left(s_{i}+z+v\right) r_{l}^{j}\left(s_{i}+z+v\right) \\
\times \lambda_{l}^{j, j-1}\left(s_{i}+z+v\right) \mathrm{d} v \mathrm{~d} z \mathrm{~d} s_{i} \\
-\int_{0}^{\infty} \prod_{h \neq i} \sum_{m=0}^{j-1} r_{h}^{m}(t) r_{i}^{j}(t) \lambda_{i}^{j, j-1}(t) \mathrm{d} t .
\end{gathered}
$$

\section{Reduction in the remaining system time above a specific state due to a jump downwards of a component inside a module}

Let the multistate strongly coherent system have the modular decomposition $\left\{M_{g}, \chi_{g}\right\}_{g=1}^{a}$, defined in the same way as in binary theory by Barlow and Proschan (1981) and in multistate theory by Natvig (2011b). We introduce the random variable $Z_{M_{g}, b, j}$, this being the fictive increase in the remaining system time above state $j$ due to a fictive minimal repair of the $g$ th module at its time of jump downwards from states $\{b, \ldots, M\}$ to $\{0, \ldots, b-1\}$, where $b \in\{1, \ldots, M\}$. Since a module consists of more than one component, we think that this minimal repair should not be of the 'black box' type. Having in mind what is going on physically, the minimal repair of the module should rather be interpreted as a 'black box' minimal repair of the component in the module that 'caused' the module to make such a jump downwards by itself jumping downwards. This was done in Natvig (1979), (1982a), and (1990), and will also be the approach in the present paper. In the multistate case we get a contribution to the distribution of 
$Z_{M_{g}, b, j}$ for all components $i \in M_{g}$ and all jumps downwards for these components from state $k$ to state $k-1$ for $k \in\{1, \ldots, M\}$.

Let

$$
Z_{M_{g}, b, j}=Y_{M_{g}, b, j}^{1}-Y_{M_{g}, b, j}^{0},
$$

where

- $Y_{M_{g}, b, j}^{1}$ is the system time in the states $\{j, \ldots, M\}$ in the interval $\left[T_{i, k-1}, T_{i, k-1}^{\prime}\right]$ just after the jump downwards from state $k$ to state $k-1$ of the $i$ th component being inside $M_{g}$, and also of $M_{g}$ jumping downwards from $\{b, \ldots, M\}$ to $\{0, \ldots, b-1\}$. The component and hence also $M_{g}$, however, immediately undergoes a fictive minimal repair.

- $Y_{M_{g}, b, j}^{0}$ is the system time in the states $\{j, \ldots, M\}$ in the interval $\left[T_{i, k-1}, T_{i, k-1}^{\prime}\right]$ just after the jump downwards from state $k$ to state $k-1$ of the $i$ th component being inside $M_{g}$, and also of $M_{g}$ jumping downwards from $\{b, \ldots, M\}$ to $\{0 \ldots, b-1\}$, assuming that the component stays in the latter state throughout this interval.

Let $I$ be the random component inside $M_{g}$ making a jump downwards from the random state $K$ to $K-1$ when $M_{g}$ is jumping downwards from $\{b, \ldots, M\}$ to $\{0, \ldots, b-1\}$. Hence, $Z_{M_{g}, b, j}=Z_{I, K, j}$.

Lemmas 3 and 4 below, given without proofs, are generalizations of a part of Theorem 2.6 in Natvig (1982a) covering a binary coherent system.

Lemma 3. It holds that

$$
\begin{aligned}
& \bar{G}_{M_{g}, b, j}^{1}(u) \\
& =\mathbb{P}\left(Y_{M_{g}, b, j}^{1}>u\right) \\
& =\sum_{i \in M_{g}}\left\{\sum _ { k \in \{ 1 , \ldots , M - 1 \} } \int _ { s _ { i } = 0 } ^ { \infty } \int _ { z = 0 } ^ { \infty } \sum _ { ( \cdot { } _ { ( M g } ) ^ { c } \cup \{ i \} } \sum _ { w } \sum _ { ( \cdot { } _ { i } , \boldsymbol { x } ^ { M _ { g } } ) \leq \boldsymbol { w } } \mathbf { 1 } \left(\chi_{g}\left(k_{i}, \boldsymbol{w}\right) \geq b,\right.\right. \\
& \left.\chi_{g}\left((k-1)_{i}, \boldsymbol{w}\right)<b\right) \\
& \times \prod_{h \in M_{g}-\{i\}}\left[r_{h}^{w_{h}}\left(s_{i}+z\right) p_{h}^{w_{h}, x_{h}}\left(s_{i}+z, s_{i}+z+u\right)\right] \\
& \times r_{i}^{k+1}\left(s_{i}\right) \lambda_{i}^{k+1, k}\left(s_{i}\right) f_{i}^{k}(z) \frac{\bar{F}_{i}^{k}(z+u)}{\bar{F}_{i}^{k}(z)} \\
& \times \sum_{\left(\cdot M_{g}, \boldsymbol{x}\right)} \mathbf{1}\left(\phi\left(k_{i}, \boldsymbol{x}\right) \geq j\right) \prod_{h \in M_{g}^{c}} r_{h}^{x_{h}}\left(s_{i}+z+u\right) \mathrm{d} z \mathrm{~d} s_{i} \\
& +\int_{z=0}^{\infty} \sum_{\left(\cdot{ }_{\left(M_{g}\right)^{c} \cup\{i\}}, w\right)} \sum_{\left({ }_{\cdot}, \boldsymbol{x}^{M_{g}}\right) \leq \boldsymbol{w}} \mathbf{1}\left(\chi_{g}\left(M_{i}, \boldsymbol{w}\right) \geq b, \chi_{g}\left((M-1)_{i}, \boldsymbol{w}\right)<b\right) \\
& \times \prod_{h \in M_{g}-\{i\}} r_{h}^{w_{h}}(z) p_{h}^{w_{h}, x_{h}}(z, z+u) f_{i}^{M}(z) \frac{\bar{F}_{i}^{M}(z+u)}{\bar{F}_{i}^{M}(z)} \\
& \left.\times \sum_{\left(\cdot M_{g}, \boldsymbol{x}\right)} \mathbf{1}\left(\phi\left(M_{i}, \boldsymbol{x}\right) \geq j\right) \prod_{h \in M_{g}^{c}} r_{h}^{x_{h}}(z+u) \mathrm{d} z\right\},
\end{aligned}
$$




$$
\begin{aligned}
& \bar{G}_{M_{g}, b, j}^{0}(v) \\
& =\mathbb{P}\left(Y_{M_{g}, b, j}^{0}>v\right) \\
& =\sum_{i \in M_{g}} \sum_{k \in\{1, \ldots, M-1\}} \int_{s_{i}=0}^{\infty} \int_{z=0}^{\infty} \sum_{\left(\cdot{ }_{(M g}\right)^{c} \cup\{i\}} \sum_{\left({ }_{i}, x^{M_{g}}\right) \leq \boldsymbol{w}} \mathbf{1}\left(\chi_{g}\left(k_{i}, \boldsymbol{w}\right) \geq b,\right. \\
& \left.\chi_{g}\left((k-1)_{i}, \boldsymbol{w}\right)<b\right) \\
& \times \prod_{h \in M_{g}-\{i\}}\left[r_{h}^{w_{h}}\left(s_{i}+z\right) p_{h}^{w_{h}, x_{h}}\left(s_{i}+z, s_{i}+z+v\right)\right] \\
& \times r_{i}^{k+1}\left(s_{i}\right) \lambda_{i}^{k+1, k}\left(s_{i}\right) f_{i}^{k}(z) \frac{\bar{F}_{i}^{k}(z+v)}{\bar{F}_{i}^{k}(z)} \\
& \times \sum_{\left(\cdot M_{g}, \boldsymbol{x}\right)} \mathbf{1}\left(\phi\left((k-1)_{i}, \boldsymbol{x}\right) \geq j\right) \prod_{h \in M_{g}^{c}} r_{h}^{x_{h}}\left(s_{i}+z+v\right) \mathrm{d} z \mathrm{~d} s_{i} \\
& +\int_{z=0}^{\infty} \sum_{\left({ }_{\left(M_{g}\right)^{c} \cup\{i\}}, w\right)} \sum_{\left({ }_{i}, \boldsymbol{x}^{M g}\right) \leq \boldsymbol{w}} \mathbf{1}\left(\chi_{g}\left(M_{i}, \boldsymbol{w}\right) \geq b, \chi_{g}\left((M-1)_{i}, \boldsymbol{w}\right)<b\right) \\
& \times \prod_{h \in M_{g}-\{i\}} r_{h}^{w_{h}}(z) p_{h}^{w_{h}, x_{h}}(z, z+v) f_{i}^{M}(z) \frac{\bar{F}_{i}^{M}(z+v)}{\bar{F}_{i}^{M}(z)} \\
& \left.\times \sum_{\left(\cdot M_{g}, \boldsymbol{x}\right)} \mathbf{1}\left(\phi\left((M-1)_{i}, \boldsymbol{x}\right) \geq j\right) \prod_{h \in M_{g}^{c}} r_{h}^{x_{h}}(z+v) \mathrm{d} z\right\} .
\end{aligned}
$$

Furthermore, for $h \neq i$ and $0 \leq t, 0 \leq v \leq u$, let

$$
p_{h, t, t+v}^{w_{h}, x_{h}, y_{h}}(t+u)=\mathbb{P}\left(X_{h}(t+u)=y_{h} \mid X_{h}(t)=w_{h}, X_{h}(t+v)=x_{h}\right) .
$$

Lemma 4. For $0 \leq v \leq u$,

$$
\begin{aligned}
& \bar{G}_{M_{g}, b, j}(u, v) \\
& =\mathbb{P}\left(Y_{M_{g}, b, j}^{1}>u, Y_{M_{g}, b, j}^{0}>v\right) \\
& =\sum_{i \in M_{g}}\left\{\sum _ { k \in \{ 1 , \ldots , M - 1 \} } \int _ { s _ { i } = 0 } ^ { \infty } \int _ { z = 0 } ^ { \infty } \sum _ { ( \cdot { } _ { ( M g } ) ^ { c } \cup \{ i \} } \sum _ { ( \cdot { } _ { i } , y ^ { M _ { g } } ) \leq ( \cdot { } _ { i } , \boldsymbol { x } ^ { M _ { g } } ) \leq \boldsymbol { w } } \mathbf { 1 } \left(\chi_{g}\left(k_{i}, \boldsymbol{w}\right) \geq b,\right.\right. \\
& \left.\chi_{g}\left((k-1)_{i}, \boldsymbol{w}\right)<b\right) \\
& \times \prod_{h \in M_{g}-\{i\}}\left[r_{h}^{w_{h}}\left(s_{i}+z\right) p_{h}^{w_{h}, x_{h}}\left(s_{i}+z, s_{i}+z+v\right)\right. \\
& \left.\times p_{h, s_{i}+z, s_{i}+z+v}^{w_{h}, x_{h}, y_{h}}\left(s_{i}+z+u\right)\right] \\
& \times \sum_{\left(\cdot M_{g}, \boldsymbol{x}\right)} \sum_{\left(\cdot M_{g}, \boldsymbol{y}\right) \leq\left(\cdot M_{g}, \boldsymbol{x}\right)} \mathbf{1}\left(\phi\left((k-1)_{i}, \boldsymbol{x}\right) \geq j\right) \\
& \times \mathbf{1}\left(\phi\left(k_{i}, \boldsymbol{y}\right) \geq j\right) \\
& \times \prod_{h \in M_{g}^{c}}\left[r_{h}^{x_{h}}\left(s_{i}+z+v\right) p_{h}^{x_{h}, y_{h}}\left(s_{i}+z+v, s_{i}+z+u\right)\right] \\
& \times r_{i}^{k+1}\left(s_{i}\right) \lambda_{i}^{k+1, k}\left(s_{i}\right) f_{i}^{k}(z) \frac{\bar{F}_{i}^{k}(z+u)}{\bar{F}_{i}^{k}(z)} \mathrm{d} z \mathrm{~d} s_{i}
\end{aligned}
$$




$$
\begin{aligned}
+\int_{z=0}^{\infty} & \left.\sum_{\left(\cdot{ }_{\left(M_{g}\right)}{ }^{c} \cup\{i\}\right.}, \boldsymbol{w}\right) \\
\quad \times & \sum_{\left(\cdot{ }_{i}, \boldsymbol{y}^{M_{g}}\right) \leq\left(\cdot{ }_{i}, \boldsymbol{x}^{M_{g}}\right) \leq \boldsymbol{w}} \mathbf{1}\left(\chi_{g}\left(M_{i}, \boldsymbol{w}\right) \geq b, \chi_{g}\left((M-1)_{i}, \boldsymbol{w}\right)<b\right) \\
& \times \sum_{h \in M_{g}-\{i\}}\left[r_{h}^{w_{h}}(z) p_{h}^{w_{h}, x_{h}}(z, z+v) p_{h, z, z+v}^{w_{h}, x_{h}, y_{h}}(z+u)\right] \\
& \times \sum_{\left(\cdot M_{g}, \boldsymbol{x}\right)} \sum_{\left(\cdot M_{g}, \boldsymbol{y}\right) \leq\left(\cdot M_{g}, \boldsymbol{x}\right)} \mathbf{1}\left(\phi\left((M-1)_{i}, \boldsymbol{x}\right) \geq j\right) \mathbf{1}\left(\phi\left(M_{i}, \boldsymbol{y}\right) \geq j\right) \\
& \left.\times \prod_{h \in M_{g}^{c}}\left[r_{h}^{x_{h}}(z+v) p_{h}^{x_{h}, y_{h}}(z+v, z+u)\right] f_{i}^{M}(z) \frac{\bar{F}_{i}^{M}(z+u)}{\bar{F}_{i}^{M}(z)} \mathrm{d} z\right\}
\end{aligned}
$$

An expression for $\mathbb{P}\left(Z_{M_{g}, b, j}=0\right)$ is given by the following theorem, again, as in Theorem 1 , it is not based on minimal cut sets containing the $i$ th component. The absolutely continuous part of the distribution of $Z_{M_{g}, b, j}$ is completely parallel to the one given in Theorem 1 but now inserting the expressions for $G_{M_{g}, b, j}^{1}(u)$ and $\bar{G}_{M_{g}, b, j}(u, v)$ from Lemmas 3 and 4.

Theorem 2. It holds that

$$
\begin{aligned}
& \mathbb{P}\left(Z_{M_{g}, b, j}=0\right) \\
& =1-\mathbb{P}\left(Z_{M_{g}, b, j}>0\right) \\
& =1-\mathbb{P}\left(Y_{M_{g}, b, j}^{1}>Y_{M_{g}, b, j}^{0}>0\right)-\mathbb{P}\left(Y_{M_{g}, b, j}^{1}>0, Y_{M_{g}, b, j}^{0}=0\right) \\
& =1-\sum_{i \in M_{g}}\left\{\sum _ { k \in \{ 1 , \ldots , M - 1 \} } \int _ { s _ { i } = 0 } ^ { \infty } \int _ { z = 0 } ^ { \infty } \int _ { v = 0 } ^ { \infty } \sum _ { ( \cdot { } _ { ( M g } ) ^ { c } \cup \{ i \} } \sum _ { w ) } \mathbf { 1 } \left(\chi_{g}\left(k_{i}, \boldsymbol{w}\right) \geq b,\right.\right. \\
& \left.\chi_{g}\left((k-1)_{i}, \boldsymbol{w}\right)<b\right) \\
& \times \prod_{h \in M_{g}-\{i\}}\left[r_{h}^{w_{h}}\left(s_{i}+z\right) p_{h}^{w_{h}, x_{h}}\left(s_{i}+z, s_{i}+z+v\right)\right] \\
& \times r_{i}^{k+1}\left(s_{i}\right) \lambda_{i}^{k+1, k}\left(s_{i}\right) f_{i}^{k}(z) \frac{\bar{F}_{i}^{k}(z+v)}{\bar{F}_{i}^{k}(z)} \\
& \times \sum_{\left(\cdot M_{g}, \boldsymbol{x}\right)} \prod_{h \in M_{g}^{c}} r_{h}^{x_{h}}\left(s_{i}+z+v\right) \\
& \times \sum_{l \neq i} \mathbf{1}\left(x_{l}>0\right) \lambda_{l}^{x_{l}, x_{l}-1}\left(s_{i}+z+v\right) \\
& \times\left[\mathbf{1}\left(\phi\left((k-1)_{i},\left(x_{l}\right)_{l}, \boldsymbol{x}\right) \geq j\right) \mathbf{1}\left(\phi\left(k_{i},\left(x_{l}-1\right)_{l}, \boldsymbol{x}\right) \geq j\right)\right. \\
& \left.-\mathbf{1}\left(\phi\left((k-1)_{i},\left(x_{l}-1\right)_{l}, \boldsymbol{x}\right) \geq j\right)\right] \mathrm{d} v \mathrm{~d} z \mathrm{~d} s_{i} \\
& +\int_{z=0}^{\infty} \int_{v=0}^{\infty} \sum_{\left(\cdot{ }_{(M g}\right)^{c} \cup\{i\}} \sum_{\left(\cdot, i, x^{M_{g}}\right) \leq \boldsymbol{w}} \mathbf{1}\left(\chi_{g}\left(M_{i}, \boldsymbol{w}\right) \geq b, \chi_{g}\left((M-1)_{i}, \boldsymbol{w}\right)<b\right) \\
& \times \prod_{h \in M_{g}-\{i\}}\left[r_{h}^{w_{h}}(z) p_{h}^{w_{h}, x_{h}}(z, z+v)\right] f_{i}^{M}(z) \frac{\bar{F}_{i}^{M}(z+v)}{\bar{F}_{i}^{M}(z)} \\
& \times \sum_{\left(\cdot M_{g}, \boldsymbol{x}\right)} \prod_{h \in M_{g}^{c}} r_{h}^{x_{h}}(z+v) \sum_{l \neq i} I\left(x_{l}>0\right) \lambda_{l}^{x_{l}, x_{l}-1}(z+v)
\end{aligned}
$$




$$
\begin{aligned}
& \times {\left[\mathbf{1}\left(\phi\left((M-1)_{i},\left(x_{l}\right)_{l}, \boldsymbol{x}\right) \geq j\right) \mathbf{1}\left(\phi\left(M_{i},\left(x_{l}-1\right)_{l}, \boldsymbol{x}\right) \geq j\right)\right.} \\
&\left.\left.-\mathbf{1}\left(\phi\left((M-1)_{i},\left(x_{l}-1\right)_{l}, \boldsymbol{x}\right) \geq j\right)\right] \mathrm{d} v \mathrm{~d} z\right\} \sum_{i \in M_{g}} \sum_{k \in\{1, \ldots, M\}} \int_{t=0}^{\infty} \sum_{(\cdot i,} \mathbf{x} \\
& \mathbf{1}\left(\chi_{g}\left(k_{i}, \boldsymbol{x}^{M_{g}}\right) \geq b, \chi_{g}\left((k-1)_{i}, \boldsymbol{x}^{M_{g}}\right)<b\right) \\
& \times\left[\mathbf{1}\left(\phi\left(k_{i}, \boldsymbol{x}\right) \geq j\right)-\mathbf{1}\left(\phi\left((k-1)_{i}, \boldsymbol{x}\right) \geq j\right)\right] \\
& \times \prod_{l \neq i} r_{l}^{x_{l}}(t) r_{i}^{k}(t) \lambda_{i}^{k, k-1}(t) \mathrm{d} t .
\end{aligned}
$$

Since $Z_{M_{g}, b, j}=Z_{I, K, j}$, it follows that $Z_{M_{g}, b, j}>0$ implies the existence of $i \in M_{g}$ and $k \in\{1, \ldots, M\}$ such that $Z_{i, k, j}>0$. The reverse implication, on the other hand, is not true. To see an example of this, let $M_{g}=\{i, m\}$ be a parallel system of two components. In this special case the module leaves the states $\{b, \ldots, M\}$ at $\max \left(T_{i, b-1}, T_{m, b-1}\right)$. Suppose that $T_{i, b-1}<T_{m, b-1}$ and that the system subsequently leaves the states $\{j, \ldots, M\}$ at $T_{l, d-1}>T_{m, b-1}$ for some $l \in M_{g}^{c}, d \in\{1, \ldots, M\}$. It may then happen that $T_{i, b-1}^{\prime}>T_{l, d-1}$, i.e. the effect of the minimal repair of component $i$ at $T_{i, b-1}$ extends beyond $T_{l, d-1}$, which again may lead to $Z_{i, b, j}>0$. At the same time, we may have $T_{m, b-1}^{\prime}<T_{l, d-1}$, in which case $Z_{M_{g}, b, j}=Z_{m, b, j}=0$.

\section{Concluding remarks}

To work out the lemmas and theorems of this paper has been challenging even when only considering nonrepairable systems. From the expressions given in Natvig (2011a) for the Natvig measure for repairable systems in a time interval $[0, t]$ based on expectations, it seems to be over the top to arrive at the corresponding distributions in an analytical form. The answer to this is to use advanced discrete event simulation methods as applied to multistate network flow systems of repairable components as in Huseby and Natvig (2013). As a first step we should work out a simulation program for the case of nonrepairable systems. To arrive at such a program, the developments and results of the present paper should be very helpful, for instance, by checking that it produces the correct results for the simple multistate series and parallel systems.

\section{Acknowledgements}

I am very grateful to my colleague Jørund Gåsemyr for extremely careful reading of earlier drafts of this manuscript, for detecting several mistakes, and for coming up with alternative approaches. For instance, he revealed that a minimal cut sets approach in Theorems 1 and 2 was not fruitful.

\section{References}

Barlow, R. E. and Proschan, F. (1975). Importance of system components and fault tree events. Stoch. Process. Appl. 3, 153-173.

Barlow, R. E. and Proschan, F. (1981). Statistical Theory of Reliability and Life Testing. Probability Models. To Begin With, Silver Springs, MD.

Birnbaum, Z. W. (1969). On the importance of different components in a multicomponent system. In Multivariate Analysis, II, Academic Press, New York, pp. 581-592.

Huseby, A. B. AND NATvig B. (2013). Discrete event simulation methods applied to advanced importance measures of repairable components in multistate network flow systems. Reliab. Eng. System Safety 119, 186-198. 
Kuo, W. And Zhu, X. (2012). Importance Measures in Reliability, Risk, and Optimization: Principles and Applications. John Wiley, Hoboken, NJ.

Natvig, B. (1979). A suggestion of a new measure of importance of system components. Stoch. Process. Appl. 9, $319-330$

Natvig, B. (1982a). On the reduction in remaining system lifetime due to the failure of a specific component. J. Appl. Prob. 19, 642-652. (Correction: J. Appl. Prob. 20, 713.)

Natvig, B. (1982b). Two suggestions of how to define a multistate coherent system. Adv. Appl. Prob. 14, 434-455.

Natvig, B. (1985). New light on measures of importance of system components. Scand. J. Statist 12, 43-54.

NATVIG, B. (1990). On information-based minimal repair and the reduction in remaining system lifetime due to the failure of a specific module. J. Appl. Prob. 27, 365-375.

NATVIG, B. (2011a). Measures of component importance in nonrepairable and repairable multistate strongly coherent systems. Methodol. Comput. Appl. Prob. 13, 523-547.

Natvig, B. (2011b). Multistate Systems Reliability Theory with Applications. John Wiley, Chichester.

NAtvig, B. AND GÅsemyr, J. (2009). New results on the Barlow-Proschan and Natvig measures of component importance in nonrepairable and repairable systems. Methodol. Comput. Appl. Prob. 11, 603-620.

RAmirez-Marquez, J. E. AND CoIt, D. W. (2007). Multi-state component criticality analysis for reliability improvement in multi-state systems. Reliab. Eng. System Safety 92, 1608-1619.

Ramirez-Marquez, J. E., et al. (2006). New insights on multi-state component criticality and importance. Reliab. Eng. System Safety 91, 894-904.

Zio, E., Marella, M. And Podofillini, L. (2007). Importance measures-based prioritization for improving the performance of multi-state systems: application to the railway industry. Reliab. Eng. System Safety 92, 1303-1314.

Zio, E., Podofillini, L. And Levitin, G. (2004). Estimation of the importance measures of multi-state elements by Monte Carlo simulation. Reliab. Eng. System Safety 86, 191-204. 